\title{
THE NILPOTENT LENGTH OF FINITE SOLUBLE GROUPS
}

\author{
Dedicated to the memory of Hanna Neumann
}

M. FRICK

(Received 28 June 1972)

Communicated by M. F. Newman

\section{Introduction}

Throughout this paper a "group" will mean a "finite soluble group".

Carter, Fischer and Hawkes [1] call a group $G$ critical for a class $\ddot{X}$ of groups if every proper subgroup of $G$, but not $G$ itself, belongs to $\mathfrak{X}$. We shall say a group is $\mathbf{Q}$-critical if it is critical for $\mathbf{Q}(h)$ for some integer $n$ (where $\mathfrak{Q}(h)$ denotes the class of groups of nilpotent length at most $n$ ). Thus a group is $\mathbb{Q}$-critical if its nilpotent length is greater than that of each of its proper subgroups.

The class of $\mathbb{L}$-critical groups is not closed under homomorphic images. For example, the group $G=A B$ where $A$ is cyclic of order $3, B$ is cyclic of order 4 , and a generator of $B$ takes a generator of $A$ to its inverse is $\mathbb{R}$-critical, whereas $G / A$ is not. Knowledge of when a homomorphic image of an $\mathbb{Q}$-critical group is $\mathbb{E}$-critical can be useful when proving results on $\mathbb{\&}$-critical groups by induction methods. The main theorem of this paper implies that a homomorphic image of an $\mathfrak{R}$-critical group is $\mathfrak{Q}$-critical if it possesses a complemented minimal normal subgroup. This result is then used in the last section of this paper, which is an attempt at giving as complete a description of the $\mathbb{L}$-critical $A$-groups as possible. ( $A$-groups are groups whose Sylow subgroups are all abelian.) The author was, however, unable to give a complete classification up to isomorphism classes of these groups.

This paper is an extract of the author's M.Sc. thesis, submitted to the Australian National University in February, 1972. The valuable assistance of Dr. John Cossey, who suggested the problems and supervised the research, is gratefully acknowledged.

\section{Main Theorem}

We first give some notation and definitions and note down some results from [1] that will be needed to prove the main theorem. 
The Frattini subgroup of a group $G$ will be denoted by $\Phi(G)$.

A chief factor $H / K$ of a group $G$ is said to be complemented in $G$ if $G$ has a subgroup $M$ such that $H M=G$ and $H \cap M \leqq K$. If no such $M$ exists, then $H / K \leqq \Phi(G / K)$ and in this case $H / K$ is said to be Frattini.

When speaking of the $k$ th complemented chief factor of a group $G$ in a chief series

$$
1=G_{0}<G_{1}<\cdots<G_{n}=G
$$

of $G$, the counting will be from left to right.

If $G$ is a group then $L(G)$ will denote the smallest normal subgroup of $G$ such that $G / L(G)$ is nilpotent. The lower nilpotent series of $G$ is the series $\left\{L_{i}(G)\right\}$ defined by

$$
L_{0}(G)=G, L_{i}(G)=L\left(L_{i-1}(G)\right), i=1,2, \cdots .
$$

The Fitting subgroup of $G$ will be denoted by $F(G)$. The upper nilpotent series $\left\{F_{i}(G)\right\}$ of $G$ is the series defined by

$$
F_{0}(G)=1, F_{,}(G) / F_{i-1}(G)=F\left(G / F_{i-1}(G)\right), i=1,2, \cdots
$$

The nilpotent length of $G$ is the smallest integer $n$ such that $L_{n}(G)=1$ (or, equivalently, $F_{n}(G)=G$ ) and will be denoted by $l(G)$.

As in [1] the series $\{\Phi,(G)\}$ is defined by

$$
\Phi_{i}(G) / F_{\imath-1}(G)=\Phi\left(G / F_{i-1}(G)\right), i=1,2, \cdots .
$$

Following [1], we say a group $G$ is extreme if $F_{i}(G) / \Phi_{i}(G)$ is a chief factor of $G$ for each $i=1,2, \cdots, l(G)$. From [1] Theorem 2.8 we have

LeMMA 2.1. Every factor group of an extreme group is extreme.

The number of comp'emented chief factors in a chief series of a group $G$ will be denoted by $c(G)$. (Lemma 2.6 of [1] shows that this number is independent of the particular chief series.) The following characterization of extreme groups is given in [1] Theorem 2.9 (iii).

LEMMA 2.2. A group $G$ is extreme if and only if $l(G)=c(G)$.

From [1] Theorem 5.1 follows

LEMMA 2.3. If $G$ is an $\mathfrak{L}$-critical group then

(a) $G$ is extreme, and

(b) $G / F(G)$ is also R-critical.

The main theorem will now be proved in the form

THEOREM 2.4. If $G$ is an $\mathfrak{L}$-critical group and $M / N$ a complemented chief factor of $G$, then $G / N$ is $\mathfrak{Q}$-critical. 
Proof. Let $\mathbb{C}$ be a chief series of $G$ through $M$ and $N$, and suppose $M / N$ is the $k$ th complemented factor of $\mathfrak{C}$. $(1 \leqq k \leqq c(G))$. If $k=1$, then $N \leqq \Phi(G)$ and then $l(G / N)=l(G)$, so in this case $G / N$ is $\mathbb{Q}$-critical since $G$ is $\mathbb{Q}$-critical.

Now let $k>1$ and assume that the theorem is true for the $(k-1)$ th complemented factor $A / B$ of $C$. Without loss of generality we may assume that $B=1$. Then $M / N$ is the second complemented factor of some chief series of $G .1$

It follows from Lemma 2.3 that $G$ as well as $G / F(G)$ are extreme. Therefore, by Lemma 2.2, every chief series of $G$ has only one complemented factor below $F(G)$. If $F(G) \cap M \equiv N$ then $F(G) \cap M / F(G) \cap N$ would be a complemented chief factor of $G$ and then $G$ would have a series with two complemented chief factors below $F(G)$, which it does not. Therefore $F(G) \cap M \leqq N$. Let $n=l(G)$. Lemma 2.1 implies that $G / M$ as well as $G / N$ are extreme so, by Lemma 2.2,

and

$$
l(G / N)=c(G / N)=n-1
$$

$$
l(G / M)=c(G / M)=n-2 .
$$

Let $K / N$ be a maximal subgroup of $G / N$. Now $N \$ \Phi(G)$ and $F(G) / \Phi(G)$ is a chief factor of $G$, so

$$
K \geqq \Phi(G) N \geqq F(G) .
$$

But $G / F(G)$ is $\mathcal{Q}$-critical by Lemma 2.3 (b), hence

so that

$$
l(K / F(G)) \leqq n-2,
$$

and hence

$$
L_{n-2}(K) \leqq F(G) \cap M \leqq N
$$

$$
l(K / N) \leqq n-2<l(G / N) .
$$

Therefore $G / N$ is $\mathfrak{L}$-critical.

\section{On $\mathfrak{L}$-critical $A$-groups}

The derived group of a group $G$ will be denoted by $G$. The derived series of $G$ is the series $\left\{G^{(i)}\right\}$ defined by

$$
G^{(0)}=G, G^{(i)}=\left(G^{(i-1)}\right)^{\prime} i=1,2, \cdots .
$$

The derived length of $\mathrm{G}$ is the smallest integer $n$ such that $G^{(n)}=1$.

In the case of an $A$-group the lower nilpotent series and the derived series coincide, and the terms "derived length" and "nilpotent length" are identical.

The following result is Lemma 5.2 of [1].

LEMMA 3.1. If $G$ is an extreme group then $L_{i-1}(G) / L_{i}(G)$ has prime power order for $i=1, \cdots, l(G)$. 
(Note that our enumeration of the terms of the lower nilpotent series is different from that in [1].)

From [3] Theorem 8.3 follows

LEMMA 3.2. If $G$ is an $A$-group then the order of $G$ is divisible by at least $l(G)$ distinct primes.

A group is said to be homocyclic if it is abelian of type $\left(p^{t}, . p^{t}, \cdots, p^{t}\right)$ for some prime power $p^{t}$.

THEOREM 3.3. Let $G$ be an A-group of derived length $n$. Then $G$ is $\mathfrak{L}$-critical if and only if the order of $G$ is divisible by exactly $n$ distinct primes and $G$ has Sylow subgroups $M_{1}, M_{2}, \cdots, M_{n}$, corresponding to these primes which have the following properties.

(i) $M_{i}$ normalizes $M_{j}$ for $i>j$.

(ii) $G^{(i)}=M_{1} M_{2} \cdots M_{n-1}$ for $i=0,1, \cdots, n-1$.

(iii) Each $M_{\imath}$ is homocyclic. Also, $M_{1}$ is elementary abelian and $M_{n}$ is cyclic.

(iv) Let $p_{i}^{t_{1}}$ be the exponent of $M_{i}(i=1, \cdots, n)$. Then the factors

$M_{1} M_{2} \cdots M_{i-1} M_{i}^{p_{i}^{k-1}} / M_{1} M_{2} \cdots M_{i-1} M_{i}^{p_{i}^{k}}, k=1, \cdots, t_{i}, i=1, \cdots, n$,

are all chief factors of $G$.

(v) $C_{M_{1+1}}\left(M_{t}\right)=\Phi\left(M_{\imath+1}\right)$ for $i=1, \cdots, n-1$.

Proof. Let $G$ be an $Q$-critical $A$-group of derived length $n$. It follows from Lemma 3.1 and Lemma 3.2 that the order of $G$ is divisible by exactly $n$ distinct primes. Therefore it follows from Lemma 3.1 that $G^{(i-1)} / G^{(i)}$ is a Sylow subgroup of $G / G^{(i)}$ and hence, by the Schur-Zassenhaus Theorem, has a complement in $G / G^{(i)}$ for $i=1, \cdots, n$. Now let $M_{1}=G^{(n-1)}$ and $H_{2}$ be a complement for $M_{1}$ in $G$, then let $M_{2}=H_{2}^{(n-2)}$ and $H_{3}$ be a complement for $M_{2}$ in $H_{2}$, let $M_{3}=H_{3}^{(n-3)}$ etc. Choosing the $M_{i}$ in this way, we see that (i) and (ii) are satisfied.

(iii) Let $i \in\{1, \cdots, n\}$. By [3] Lemma $6.1, M_{i}$ can be written as

$$
M_{i}=A_{1} \times A_{2} \times \cdots \times A_{r}
$$

where each $A_{i}$ is an indecomposable homocyclic $H_{i+1}$-group. (Take $H_{n+1}=1$.) By [3] Corollary 6.2, each factor $A_{j} / A_{j}^{p_{i}}$ (where $p_{i}$ is the prime divisor of the order of $M_{i}$ ) is an irreducible $H_{i-1}$-group, and hence the factors

$$
M_{1} M_{2} \cdots M_{i+1} A_{j} / M_{1} M_{2} \cdots M_{1-1} A_{j}^{p_{i}}, j=1, \cdots, r
$$


are all complemented chief factors of $G$. However, it follows from Lemma 2.2 that every chief series of $G$ has only one complemented factor between $M_{1} M_{2} \ldots$ $M_{i-1}$ and $M_{1} M_{2} \cdots M_{i-1} M_{i}$. Therefore $r=1$ and hence $M_{i}$ is an indecomposable homocyclic $H_{i+1}$-group. As we have taken $H_{n+1}=1$, it follows that $M_{n}$ is cyclic.

Let $q=p_{1}^{t_{1}}-1$. Then the map $\theta: M_{1} \rightarrow M_{1}^{q}$ defined by

$$
m \theta=m^{q} \text { for all } m \in M_{1}
$$

is an $H_{2}$-homomorphism of $M_{1}$ onto $M_{1}^{q}$ with kernel $M_{1}^{p_{1}}$, so $M_{1} / M_{1}^{p_{1}}$ is $H_{2^{-}}$ isomorphic to $M_{1}^{q}$, and hence $G / M_{1}^{p_{1}}$ is isomorphic to $M_{1}^{q} M_{2} \cdots M_{n}$. But

$$
M_{1}^{p_{1}}=\Phi\left(M_{1}\right) \leqq \Phi(G),
$$

so $l\left(G / M_{1}^{p_{1}}\right)=n$. But $G$ is $\mathcal{Q}$-critical, so $t_{1}=1$ and hence $M_{1}$ is elementary abelian.

(iv) It was shown in (iii) that each $M_{i}$ is an indecomposable homocyclic $H_{i+1^{-}}$ group. Therefore it follows from [3] Corollary 6.2 that each factor

$$
M_{i}^{p_{i}^{k-1}} / M_{i}^{p_{i}^{k}}, \quad k=1, \cdots, t_{i},
$$

is an irreducible $H_{i+1}$-group, and (iv) follows.

(v) By (iv) the factors

$$
M_{1} M_{2} \cdots M_{i-1} M_{1} / M_{1} M_{2} \cdots M_{i-1} \Phi\left(M_{i}\right), i=1, \cdots, n,
$$

are all complemented chief factors of $G$. Therefore, in view of Theorem 2.4 and the fact that

$$
\mathrm{C}_{M_{i}}\left(M_{i-1}\right)=C_{M_{i}}\left(M_{i-1} / \Phi\left(M_{i-1}\right)\right), i=2, \cdots, n,
$$

(see [2] Theorem 5.1.4), it is sufficient to show that $C_{M_{2}}\left(M_{1}\right)=\Phi\left(M_{2}\right)$. But $G / F(G)$ is I-critical by Lemma 2.3 (b) so (iii) implies that $M_{2} F(G) / F(G)$ is elementary abelian. Therefore

$$
M_{2}^{p_{2}} \leqq F(G) \leqq C_{G}\left(M_{1}\right),
$$

so $\Phi\left(M_{2}\right) \leqq C_{M_{2}}\left(M_{1}\right)$. But $M_{2} / \Phi\left(M_{2}\right)$ is a chief factor of $H_{2}$, so $C_{M_{2}}\left(M_{1}\right)=\Phi\left(M_{2}\right)$.

Conversely, suppose $G$ is an $A$-group of derived length $n$ which satisfies the conditions of the theorem. Let $K$ by a maximal subgroup of $G$. Let $i$ be the smallest integer such that $G^{(i)} \$ K$ and set $H=M_{n-i} M_{n-i+1} \cdots M_{n}$. Then $H \cap K$ is a maximal subgroup of $H$, so

$$
\Phi\left(M_{n-i}\right) \leqq \Phi(H) \leqq K .
$$

It follows from (ii) and (iv) that $G^{(i)} / G^{(i+1)} \Phi\left(M_{n-i}\right)$ is a minimal normal subgroup of $G / G^{(i+1)} \Phi\left(M_{n-i}\right)$, so $K / G^{(i+1)} \Phi\left(M_{n-i}\right)$ complements $G^{(i)} / G^{(i+1)} \Phi\left(M_{n-i}\right)$ in $G / G^{(i+1)} \Phi\left(M_{n-i}\right)$. Hence 


$$
K^{(i)} \leqq K \cap G^{(i)} \leqq G^{(l+1)} \Phi\left(M_{n-i}\right)
$$

But

$$
l\left(G^{(i+1)} \Phi\left(M_{n-i}\right)\right)<n-i
$$

by (v). Therefore $l(K)<n$, and hence $G$ is $\mathfrak{Q}$-critical.

\section{References}

[1] R. W. Carter, B. Fischer and T. O. Hawkes, 'Extreme classes of finite soluble groups', J. Algebra 9 (1968), 285-313.

[2] D. G. Gorenstein, Finite Groups (Harper's Series on Modern Mathematics. Harper and Row, New York, Evanston, London, 1968.),

[3] D. R. Taunt, 'On A-groups', Proc. Cambridge Philos. Soc. 45 (1949), 14-42.

Australian National University

Canberra, A.C.T. 2600 